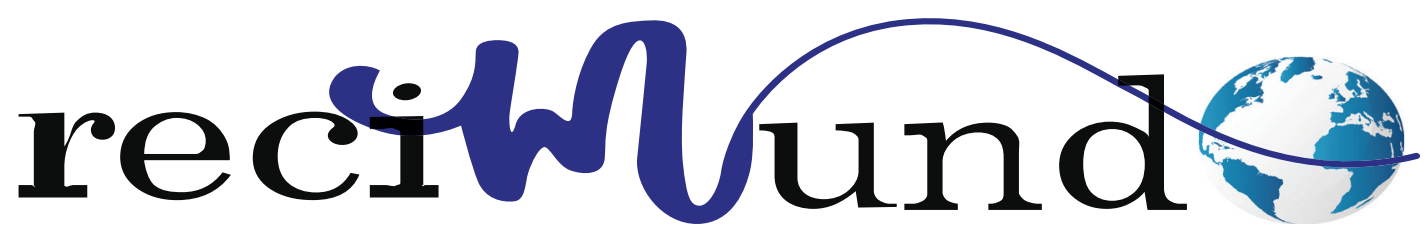

Revista Científica Mundo de la Investigación y el Conocimiento

DOI: 10.26820/recimundo/5.(1).enero.2021.305-312

URL: http://recimundo.com/index.php/es/article/view/1013

EDITORIAL: Saberes del Conocimiento

REVISTA: RECIMUNDO

ISSN: 2588-073X

TIPO DE INVESTIGACIÓN: Artículo de Revisión

CÓdigo UNESCO: 32 Ciencias Médicas

PAGINAS: 305-312

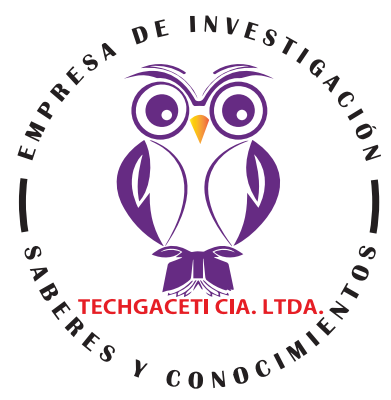

\title{
Causas y consecuencias del cáncer pulmonar
}

Causes and consequences of lung cáncer

Causas e consequências do cancro do pulmão

María Cristina Menéndez Palacios'; Genesis Denisse Mera Chapi2; José Ignacio Vera Zamora3; Ana Isabel Zambrano Mendoza ${ }^{4}$

RECIBIDO: 28/11/2020 ACEPTADO: 06/12/2020 PUBLICADO: 31/01/2021

1. Médica Cirujana; Hospital General Oskar Jandl; Galápagos, Ecuador; cristina1992@hotmail.com; https://orcid.org/00000002-3645-3840

2. Médica General; Hospital General Oskar Jandl; Galápagos, Ecuador; Denissegmera@hotmail.com; https://orcid.org/00000001-6639-3925

3. Doctor en Medicina; Hospital General Oskar Jandl; Galápagos, Ecuador; Josevera_z@hotmail.com; https://orcid.org/00000002-9504-6903

4. Médica Cirujana; Hospital General Portoviejo; Portoviejo, Ecuador; mauserrat_87@hotmail.com; https://orcid.org/00000002-8003-2349

CORRESPONDENCIA

María Cristina Menéndez Palacios

cristina1992@hotmail.com

Galápagos, Ecuador

() RECIMUNDO; Editorial Saberes del Conocimiento, 2021 


\section{RESUMEN}

El cáncer pulmonar es una de las enfermedades que más cobran vida año a año en el mundo, y está directamente asociada al tabaquismo, sin embargo la contaminación atmosférica también puede ser causante de enfermedades pulmonares crónicas que a largo pudieran desarrollar cáncer de pulmón, también los fumadores pasivos, tienen atas probabilidades de desarrollar cáncer pulmonar al estar en contacto con fumadores. La metodología de la investigación es una revisión bibliográfica. Aparte de las consecuencias de desarrollar cáncer del pulmón por efecto del cigarro, están las asociadas a los padecimientos que deben enfrentar el paciente por la sintomatología y el tipo de tratamiento que se vaya a emplear, dependiendo del estadio en que el cáncer este.

Palabras clave: Tabaquismo, cáncer, pulmón, diagnostico, nicotina.

\section{ABSTRACT}

Lung cancer is one of the diseases that occurs the most every year in the world, and is directly associated with smoking, however air pollution can also be the cause of chronic lung diseases that could develop lung cancer in the long run, also Passive smokers have a high probability of developing lung cancer from being in contact with smokers. The research methodology is a bibliographic review. Apart from the consequences of developing lung cancer due to smoking, there are those associated with the conditions that the patient must face due to the symptoms and the type of treatment that is going to be used, depending on the stage in which the cancer is.

Keywords: Smoking, cancer, lung, diagnosis, nicotine.

\section{RESUMO}

O cancro do pulmão é uma das doenças que mais ocorre todos os anos no mundo, e está directamente associado ao tabagismo, contudo a poluição do ar também pode ser a causa de doenças pulmonares crónicas que podem desenvolver cancro do pulmão a longo prazo, também os fumadores passivos têm uma elevada probabilidade de desenvolver cancro do pulmão por estarem em contacto com fumadores. A metodologia de investigação é uma revisão bibliográfica. Para além das consequências do desenvolvimento de cancro do pulmão devido ao tabagismo, existem as associadas às condições que o paciente deve enfrentar devido aos sintomas e ao tipo de tratamento que vai ser utilizado, dependendo da fase em que o cancro se encontra.

Palavras-chave: Fumar, Cancro, Pulmão, Diagnóstico, Nicotina. 


\section{Introducción}

El cáncer de pulmón no sólo es la primera causa de muerte por cáncer, es además la más evitable, ya que la mayoría de los casos de este cáncer son secundarios al tabaquismo. El cáncer de pulmón es una epidemia que, como otras, tiene una etiología y un vector. Los reportes de cáncer de pulmón a fines del siglo XIX eran anecdóticos. Todavía durante las primeras décadas del siglo pasado la incidencia era de tres casos por cada 100 000, y aumentó de manera dramática en la década de los setenta a 446 por cada 100000 hombres entre 55 y 74 años de edad. Este incremento no tiene par en otras epidemias secundarias a la conducta humana (Zinser-Sierra, 2020, págs. 303-304).

Se estima que si los hábitos de consumo no varían, en el año 2020 la mortalidad atribuible al tabaco superará los ocho millones de muertes anuales. Según la Organización Mundial de la Salud (OMS), en la actualidad existen 1100 millones de fumadores en el mundo, lo que supone un tercio de la población mundial mayor de 15 años (Botell, Bermúdez, García, \& Pieiga, 2006).

El cáncer pulmonar es la primera causa de mortalidad por cáncer en todo el mundo. Como lesión maligna es la más frecuente y letal, de comportamiento clínico muy agresivo sin respuesta a tratamiento alguno. La sobrevida a los 5 años oscila entre $5 \%$ y 16 $\%$, según corresponda a países en desarrollo o desarrollados (Martín Piñate, 2006).

Existe una relación directa entre el tabaco y el cáncer del pulmón ampliamente conocido. El $85 \%$ de los carcinomas de pulmón se da en fumadores y el riesgo aumenta proporcionalmente al número de cigarrillos que consumen al día y la profundidad de la inhalación aumenta en aquellos que fuman más de 20 a 30 cigarrillos por día. Los fumadores pasivos tienen un riesgo menor asociándose un $25 \%$ al cáncer pulmonar
(Gámez Salmón, 2015).

En estudios que han analizado la sobrevida de los pacientes con cáncer de pulmón se ha determinado que a un año del diagnóstico es de $20 \%$ y a los 5 años de $5 \%$. Para los casos con tumor extirpable quirúrgicamente puede aumentar a $35 \%$ a los 5 años. Al mirar la sobrevida según estadio al momento del diagnóstico, en un estudio se señala que a los 5 años éste fue de $65 \%$ en el estadio I, $16 \%$ en el II y en el estadio III de 19\%, mientras que en otro, las cifras obtenidas señalan que a los 5 años los pacientes en estadio IA tendrían un $61 \%$ de sobrevida, $38 \%$ en IB, en IIA $34 \%$ y $24 \%$, los con IIB, cifras que descienden a 23, 7 y $1 \%$ cuando los estadios son III A, III B y IV, respectivamente. Así también al determinar la diferencia de sobrevida en pacientes que mantuvieron el hábito tabáquico versus no fumadores, se observó que el $16 \%$ de los fumadores sobrevivieron a los 5 años, en cambio el $23 \%$ de los que nunca fumaron vivieron más allá de 5 años, 9 planteándose que el cigarro es además un fuerte predictor de sobrevida en pacientes con cáncer pulmonar (Erazo Bahamondes, 2016).

El Cáncer de Pulmón es el tumor maligno más frecuente en el tórax. De acuerdo a la clasificación de la OMS hay cuatro tipos histológicos principales:

- Carcinoma epitelial de células planas o escamosas $(30-40 \%)$

- Adenocarcinoma (25 - 30\%)

- Carcinoma de células gigantes (menos del 10\%)

Carcinoma de células pequeñas ( 15 al 20\%) Dentro de estos 4 tipos podemos diferenciar un sin número de subtipos. A considerar es el carcinoma bronquio alveolar (sinónimo de carcinoma celular alveolar), un subtipo infrecuente de adenocarcinoma que crece a partir de los alveolos. El cáncer pulmonar puede subdividirse de acuerdo a criterios diferentes y de acuerdo a su conducta bio-

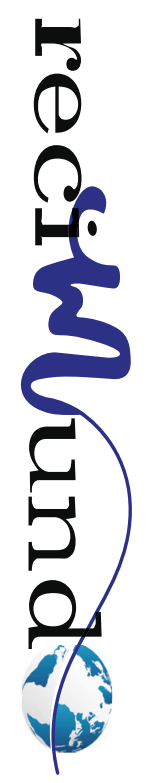


lógica y sus consecuencias en el tratamiento y pronostico, se pueden diferenciar el cáncer de pulmón de células pequeñas (15 al 20\%) y el cáncer de pulmón de células no pequeñas (Gámez Salmón, 2015).

\section{Metodología}

La metodología de la investigación, es una revisión bibliográfica, apoyada por medios electrónicos como fuente primaria de la obtención de la información sobre la temática de estudio. Se han encontrado múltiples trabajos que abordan el cáncer pulmonar y dan importantes nociones sobre las causas y consecuencias, que sirven de punto de partida para extraer las partes más relevantes y de esta manera crear un trabajo que pueda sintetizar de manera clara tan importante tema y que ayude a futuras investigaciones, sobre la materia.

\section{Resultados}

\section{Causas}

Múltiples factores carcinógenos y promotores tumorales derivados principalmente de los alquitranes, como el benzopireno y la nicotina, presentan implicaciones directas a través de sus metabolitos en la patogenia del carcinoma de pulmón. La OMS ha clasificado 107 sustancias, mezclas y situaciones de exposición como carcinógenas para el ser humano. Una de cada diez muertes del cáncer de pulmón está estrechamente relacionada con riesgos presentes en el lugar de trabajo (Peralta Nuñez, 2019).

El consumo de cigarrillo o también llamado tabaco, había sido establecido como la causa más común de cáncer de pulmón y en determinados casos, como la única, pero en el año 2013 la IARC sorprendió a la comunidad científica al establecer a la contaminación atmosférica o del aire como factor causal de cáncer pulmonar, en otras palabras "las personas que tienen mayor exposición a las partículas u contaminantes en el aire, tendrán mayor probabilidad de desarrollar cáncer de pulmón "y a su vez, según la Agencia se estimó que en el año 2010 "el cáncer pulmonar, ocasionado por la contaminación del aire, causó la muerte de 223.000 personas" (Peralta Nuñez, 2019).

Tabla 1. Factores de riesgo del Cáncer Pulmonar

\begin{tabular}{|c|c|}
\hline $\begin{array}{l}\text { Factor de } \\
\text { riesgo }\end{array}$ & Datos \\
\hline $\begin{array}{l}\text { Tabaquismo y } \\
\text { exposición al } \\
\text { humo de } \\
\text { segunda mano. }\end{array}$ & $\begin{array}{l}\text { - El humo del cigarrillo contiene más de sesenta reconocidos } \\
\text { cancerígenos } \\
\text { - Los fumadores tienen un riesgo entre } 15 \text { y } 25 \text { veces mayor } \\
\text { que los no fumadores de padecer CP } \\
\text { - La exposición pasiva al humo del tabaco incrementa el } \\
\text { riesgo de desarrollar un CP entre un } 20 \text { y un } 30 \% \text {. }\end{array}$ \\
\hline $\begin{array}{l}\text { Combustión de } \\
\text { biomasa }\end{array}$ & $\begin{array}{l}\text { - Se ha documentado con estudios epidemiológicos, la } \\
\text { vinculación entre el humo de leña doméstico y el CP. } \\
\text { - La combustión de biomasa libera partículas como fenoles, } \\
\text { acroleína, cresoles, acetaldehído, benceno, formaldehido. }\end{array}$ \\
\hline $\begin{array}{l}\text { Exposición al gas } \\
\text { radón }\end{array}$ & $\begin{array}{l}\text { Es un cancerigeno y se produce cuando el uranio se } \\
\text { descompone de manera natural en el suelo, piedras y agua. }\end{array}$ \\
\hline $\begin{array}{l}\text { Exposición al } \\
\text { asbesto }\end{array}$ & $\begin{array}{l}\text { La exposición laboral al asbesto y a otras sustancias que se } \\
\text { demostró que producen cáncer (como el arsénico, el cromo y } \\
\text { el níquel) }\end{array}$ \\
\hline $\begin{array}{l}\text { Antecedentes } \\
\text { familiares }\end{array}$ & $\begin{array}{l}\text { Las personas con un padre, hermano o hijo con cáncer de } \\
\text { pulmón tienen un mayor riesgo de padecer }\end{array}$ \\
\hline
\end{tabular}

Fuente: (Peralta Nuñez, 2019) 
La contaminación atmosférica mediante los agentes contaminantes que lo componen son causantes de una serie de enfermedades y complicaciones de alta gravedad en la salud del ser humano. Por lo tanto, la contaminación del aire puede afectar el estado de salud de una persona a través de una serie de factores o variables a las que estas se encuentren expuestas, siendo uno de los casos la alta presencia de ozono troposférico en grandes ciudades producto de la elevada presencia vehicular y la exposición crónica a este agente patógenos por grupos vulnerables como ancianos los que pueden desarrollar algún padecimiento en las vías respiratorias o en un caso diferente, la elevada tasa de combustión de biomasa en áreas rurales y la constante exposición, tanto por adultos como por niños, a este proceso, el que puede llevar a el desarrollo de afecciones como en el caso de la "Enfermedad Pulmonar Obstructiva Crónica" o también denominado como EPOC o casos de asma y sus crisis en consecuencia (Peralta Nuñez, 2019).

Tabla 2. Enfermedades según agentes contaminantes del aire

\begin{tabular}{|ll|}
\hline \multicolumn{1}{|c|}{ Agente } & \multicolumn{1}{c|}{ Enfermedades } \\
\hline Ozono troposférico & $\begin{array}{l}\text { Asma } \\
\text { Bronquitis } \\
\text { Enfisema pulmonar }\end{array}$ \\
Material particulado & $\begin{array}{l}\text { Enfermedad cardiaca } \\
\text { Accidente cerebrovascular } \\
\text { Cáncer de pulmón } \\
\text { Enfermedad pulmonar obstructiva crónica } \\
\text { Infecciones respiratorias } \\
\text { Hiperreactividad de las vías aéreas } \\
\text { Bronquitis aguda } \\
\text { Disminución de la capacidad vital de los pulmones } \\
\text { Enfisema pulmonar }\end{array}$ \\
\hline Dióxidóngeno de azufre & $\begin{array}{l}\text { Inflamación de las vias aéreas } \\
\text { Bronquitis crónica } \\
\text { Broncoconstricción }\end{array}$ \\
\hline Monóxido de carbono & Envenenamiento y muerte \\
\hline
\end{tabular}

Fuente: (Peralta Nuñez, 2019)

\section{Diagnostico}

El diagnóstico inicial de sospecha de los cánceres de pulmón se realiza mediante una radiografía del tórax. El carcinoma epidermoide y el de células pequeñas suelen verse como grandes masas en la zona central del pulmón, mientras que el adenocarcinoma y el carcinoma de células grandes suelen afectar a la zona periférica del pulmón, en ocasiones con afectación de la pleura. La confirmación de que existe una tumoración, su tamaño, sus características y el grado de extensión local se realiza mediante un TAC del tórax (www.redaccionmedica.com, 2020).
La confirmación definitiva del tipo de tumor se establece con una biopsia de pulmón obtenida mediante fibrobroncoscopia o mediante una punción de la masa a través de la piel (PAAF) y dirigida por TAC. A veces el diagnóstico se establece al tomar una biopsia de un ganglio que esté afectado y que puede encontrarse debajo de la piel o en el mediastino, en este último caso haciendo una mediastinoscopia. El diagnóstico también puede realizarse cuando existe un derrame pleural, buscando células malignas en el líquido del derrame obtenido mediante toracocentesis (www.redaccionmedica. com, 2020). 


\section{Consecuencias}

La historia natural del carcinoma del pulmón comienza con atipias celulares epiteliales bronquiales que preceden al cuadro clínico, únicamente detectados por citología o biopsia. Menos del 10\% de los carcinomas del pulmón son detectados precozmente. Con el progreso del carcinoma in situ a invasión de la pared bronquial, aparecen las primeras manifestaciones clínicas. La tos es la más frecuente. La hemóptisis se presenta en el 50\% de los casos (Gámez Salmón, 2015).

Los signos y síntomas varían con la extensión de la enfermedad, se relacionan estrechamente con la localización, el tamaño del tumor primario, la estirpe histológica y la presencia o no de metástasis. Cuando progresa el cuadro, puede aparecer un dolor pleurítico por infiltración o derrame pleural, aunque el neumotórax espontaneo puede ser la presentación inicial del carcinoma de pulmón ya sea por invasión directa o por atrapamiento de aire (Gámez Salmón, 2015).

Los signos y síntomas del CP que se manifiestan, suelen estar ligados al tipo de

cáncer que se padezca y en otras pueden mostrarse en independencia de este, "existe la posibilidad de que el cáncer pulmonar denominado incipiente no manifieste ninguna sintomatología pero fuera de ello los síntomas comunes que el CP suele manifestar pueden llegar a ser:

- Tos crónica

- Hemoptisis

- Disnea persistente

- Dolor precordial

- Sonidos pulmonares anormales "crepitaciones o estertores"

- Disminución de peso sin causa aparente

- Cefalea

- Dolor óseo (Peralta Nuñez, 2019).

El cáncer pulmonar se disemina por vía directa, linfática y hematógena. La diseminación dependerá del tipo tumoral y de localización de la lesión. El hígado y las glándulas suprarrenales son los sitios de diseminación a distancia más comunes, así como las metástasis al sistema nervioso central (Gámez Salmón, 2015).

\section{Tratamiento}

Tabla 3. Tipo de Tratamiento

\begin{tabular}{|c|l|}
\hline Tipo de tratamiento & \multicolumn{1}{|c|}{ Descripción } \\
\hline Tratamiento quirúrgico & $\begin{array}{l}\text { La cirugía es la primera opción de tratamiento, la más } \\
\text { eficaz, en los cánceres no microcíticos en estadios } \\
\text { localizados. El tipo de cirugía dependerá del tamaño, } \\
\text { localización y extensión del tumor. Cuando se extirpa un } \\
\text { lóbulo pulmonar (cada una de las partes en las que está } \\
\text { dividido el pulmón) se llama lobectomía, cuando lo que } \\
\text { se extirpa es todo un pulmón hablamos } \\
\text { de neumonectomía. }\end{array}$ \\
$\begin{array}{ll}\text { En ocasiones se puede otro tipo de cirugías como } \\
\text { la segmentectomía (se extirpa parte de un lóbulo). }\end{array}$ \\
\hline Radioterapia & $\begin{array}{l}\text { La radioterapia es el empleo de radiaciones ionizantes } \\
\text { para el tratamiento local o locorregional de determinados } \\
\text { tumores. } \\
\text { Su objetivo es destruir las células tumorales causando el } \\
\text { menor daño posible a los tejidos sanos que rodean dicho } \\
\text { tumor. El tipo de radioterapia utilizada en el cáncer de } \\
\text { pulmón es la externa, aunque pueden existir } \\
\text { excepciones. Se emplean máquinas de gran tamaño } \\
\text { (aceleradores lineales), que en ningún momento } \\
\text { contactan con el enfermo. }\end{array}$ \\
\hline
\end{tabular}




\begin{tabular}{|c|c|}
\hline Quimioterapia & $\begin{array}{l}\text { A los fármacos utilizados en este tipo de tratamiento se } \\
\text { les denomina fármacos antineoplásicos o } \\
\text { quimioterápicos. Estos fármacos llegan a prácticamente } \\
\text { todos los tejidos del organismo y ahí es donde ejercen } \\
\text { su acción tanto sobre las células malignas como sobre } \\
\text { las sanas. Debido a la acción de los medicamentos } \\
\text { sobre estas últimas, pueden aparecer una serie de } \\
\text { síntomas más o menos intensos y generalmente } \\
\text { transitorios, denominados efectos secundarios. } \\
\text { En los pacientes con estadio IV o avanzado, pacientes } \\
\text { con metástasis, es muy importante que antes de iniciar } \\
\text { el tratamiento se analicen una serie de alteraciones } \\
\text { moleculares que pueden estar presentes o no en el } \\
\text { tumor y que son necesarios para decidir la actitud } \\
\text { terapéutica. } \\
\text { En los pacientes con tumores en estadio IIIB, e se } \\
\text { considera que el pronóstico y la evolución es similar a la } \\
\text { de aquellos con enfermedad extratorácica. }\end{array}$ \\
\hline Otros tratamientos & $\begin{array}{l}\text { Láser intrabronquial: Cuando el desarrollo del tumor es } \\
\text { intrabronquial, es decir dentro del conducto del } \\
\text { bronquio, puede obstaculizar el paso del aire } \\
\text { dificultando gravemente la respiración. En estos casos } \\
\text { puede tratarse la lesión con láser, para destruir parte del } \\
\text { tumor, permitir el paso del aire, y disminuir así el ahogo } \\
\text { del paciente. El láser se aplica con la ayuda de un } \\
\text { endoscopio introducido en las vías respiratorias. } \\
\text { Prótesis endobronquiales: Esta técnica, como la } \\
\text { anterior, se utiliza cuando el desarrollo del tumor ocupa } \\
\text { el interior de los bronquios impidiendo el paso del aire. } \\
\text { Consiste en colocar mediante endoscopia una especie } \\
\text { de tubo más o menos rígido a la altura de la zona del } \\
\text { bronquio obstruida por el tumor, con el objetivo de } \\
\text { facilitar la respiración del paciente. } \\
\text { Radiofrecuencia: En los últimos años han aparecido } \\
\text { técnicas de ablación con catéter para lesiones únicas } \\
\text { pulmonares en pacientes inoperables. Esta técnica } \\
\text { consiste en quemar la lesión mediante un catéter que se } \\
\text { introduce en el pulmón. Suele usarse en tumores } \\
\text { menores de } 4 \text { cm y todavía no es una técnica estándar } \\
\text { de tratamiento. }\end{array}$ \\
\hline
\end{tabular}

Fuente: (www.aecc.es, 2020)

\section{Conclusiones}

En los últimos años han aparecido otras causas que pueden desarrollar en una persona el cáncer de pulmón, y esa es la contaminación atmosférica. La ecuación común es que interviene el humo o smog como factor determinante, que pueden causar enfermedades respiratorias crónicas y que pueden derivar en cáncer de pulmón.

Sin embargo la causa mas estudiada y directa que lo produce es el tabaquismo (cigarrillos), la frecuencia al fumar, la cantidad diaria de cigarrillos y el tiempo fumando es la mayor consecuencia que puede derivar en un cáncer pulmonar. El daño producido por el cigarro puede afectar incluso a los no fumadores (fumadores pasivos) desarrollándole enfermedades respiratorias crónicas y cáncer de pulmón a largo plazo de no evitar el humo del cigarrillo.

Aparte de la consecuencia de contraer cáncer de pulmón, la sintomatología que esta produce disminuye la calidad de vida de quien la padece, a su vez los diferentes tratamientos que se pueden emplear dependiendo del estadio en que se encentre el cáncer, pueden producir reacciones secun-

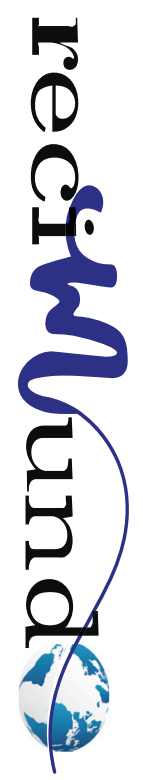


darias o daños colaterales que disminuyen aún más la calidad de vida.

\section{Bibliografía}

Botell, M. L., Bermúdez, M., García, L., \& Pieiga, E. (2006). Las consecuencias del tabaquismo. Revista Cubana de Higiene y Epidemiología, 44(3).

Erazo Bahamondes, M. (2016). Rol del tabaquismo en cancer pulmonar y dos de sus tipos histologicos.

Gámez Salmón, C. A. (2015). Descripcion de los hallazgos tomográficos en pacientes con diagnóstico histopatológico de cáncer pulmonar.

Martín Piñate, F. (2006). Pasado, presente y futuro del cáncer pulmonar. Revista de la Facultad de Medicina, 29(1), 23-28.
Peralta Nuñez, F. M. (2019). Contaminación atmosférica y su relación en el desarrollo de Cáncer de Pulmón.

www.aecc.es. (09 de 02 de 2020). Obtenido de https://www.aecc.es/es/todo-sobre-cancer/tipos-cancer/cancer-pulmon/secuelas-tratamientos-cancer-pulmon

www.redaccionmedica.com. (09 de 02 de 2020). Obtenido de https://www.redaccionmedica.com/ recursos-salud/diccionario-enfermedades/cancer-pulmon

Zinser-Sierra, J. W. (2020). Tabaquismo y cáncer de pulmón. salud pública de méxico, 61, 303-307. doi:https://doi.org/10.21149/10088

\section{CITAR ESTE ARTICULO:}

Menéndez Palacios, M. C., Mera Chapi, G. D., Vera Zamora, J. I., \& Zambrano Mendoza, A. I. (2021). Causas y consecuencias del cáncer pulmonar. RECIMUNDO, 5(1), 305-312. https://doi.org/10.26820/recimundo/5.(1).enero.2021.305-312 